\title{
Robust quantification of the $S M N$ gene copy number by real-time TaqMan PCR
}

\author{
Ilsa Gómez-Curet • Karyn G. Robinson • \\ Vicky L. Funanage • Thomas O. Crawford • \\ Mena Scavina $\cdot$ Wenlan Wang
}

Published online: 24 February 2009

(C) Springer-Verlag 2009

Erratum to: Neurogenetics (2007) 8:271-278

DOI 10.1007/s10048-007-0093-1

In the published original article, Table 1, there is an error in the sequence of the SMN probe. Letter "C" in the sixth position should be changed to "A".

The correct sequence should read:

FAM-ACA AAA TCA AAA AGA AGG AAG GTG CTC ACA-MGBNFQ.

The online version of the original article can be found at http://dx.doi. org/10.1007/s10048-007-0093-1.

I. Gómez-Curet • K. G. Robinson • V. L. Funanage • M. Scavina •

W. Wang $(\square)$

Nemours Biomedical Research,

Alfred I. duPont Hospital for Children,

P.O. Box 269, Wilmington, DE 19899, USA

e-mail: wwang@nemours.org

V. L. Funanage $\cdot$ W. Wang

Department of Pediatrics, Thomas Jefferson University,

Philadelphia, PA, USA

T. O. Crawford

Departments of Neurology and Pediatrics,

Johns Hopkins University,

Baltimore, MD, USA 
Table 1 PCR primers, TaqMan probes, and nonextending oligonucleotides (blockers) for SMN1, SMN2, and CFTR

\begin{tabular}{lll}
\hline Component & Sequence & Number of bases \\
\hline Primers & & 26 \\
SMN1-ex7F-3g & TTC CTT TAT TTT CCT TAC AGG GTg TC $^{\mathrm{a}}$ & 26 \\
SMN2-ex7F-3g & TTC CTT TAT TTT CCT TAC AGG GTg TT & 26 \\
SMN-ex7R & GCT GGC AGA CTT ACT CCT TAA TTT AA & 25 \\
CFTR-F & TAG GAA GTC ACC AAA GCA GTA CAG C & 25 \\
CFTR-R & AGC TAT TCT CAT CTG CAT TCC AAT G & 30 \\
Probes & & 29 \\
SMN probe & FAM-ACA AAA TCA AAA AGA AGG AAG GTG CTC ACA-MGBNFQ & \\
CFTR probe & VIC-TAT GAC CCG GAT AAC AAG GAG GAA CGC TC-MGBNFQ & 33 \\
Blockers & & 33 \\
SMN1 blocker & ATT TTC CTT ACA GGG TTT CAG ACA AAA TCA AAA-PO 4 & \\
SMN2 blocker & ATT TTC CTT ACA GGG TTT TAG ACA AAA TCA AAA-PO 4 & \\
\hline
\end{tabular}

${ }^{a}$ The SMN forward primers distinguished between SMN1 and SMN2 by ending on the nucleotide difference (C/T) at position 6 in exon 7 .

${ }^{\mathrm{b}} \mathrm{A}$ mismatch $\mathrm{T} \rightarrow \mathrm{G}$ was added at the -3 position from the $3^{\prime}$ end of both SMN1 and SMN2 forward primers to achieve allele specificity. 